\title{
Frequency discrimination between and within line gratings by dynamic touch
}

\author{
HAROLD T. NEFS, ASTRID M. L. KAPPERS, and JAN J. KOENDERINK \\ Universiteit Utrecht, Helmholtz Instituut, Utrecht, The Netherlands
}

\begin{abstract}
Line gratings were used to investigate the tactual discrimination thresholds for line frequency. In Experiment 1, participants were asked to discriminate between two gratings, each with a different line frequency. We used four standard frequencies in the eightfold range from 0.5 to 4 lines $/ \mathrm{cm}$. Thresholds were found to be constant at about $10.6 \%$. In this experiment, we also measured hand speed and contact force. Hand speed was roughly in the range between 0.12 and $0.44 \mathrm{~m} / \mathrm{sec}$; contact force ranged from 0.62 to $2.76 \mathrm{~N}$. In Experiment 2, we determined discrimination thresholds for line frequency transitions within a single grating. We used two frequencies and three transition lengths. The transition length had no effect on the threshold. In a third experiment, line frequency was modulated periodically. Varying the standard frequency and the size of the modulation period was found to have no effect on the discrimination thresholds. We conclude three things. First, Weber fractions for line frequency discrimination decrease as a function of line frequency within the experimental range. Second, discrimination thresholds are not altered by the length of the transition between two adjacent gratings with different line frequencies. And finally, the size of a modulation period in periodically modulated gratings is of no influence on the modulation detection threshold.
\end{abstract}

So far, only a few authors have investigated how well properties of real surfaces, such as spatial frequency or element orientation, can be discriminated or detected by touch. Most of them have addressed discrimination between two separate textures (e.g., Miyaoka, Mano, \& Ohka, 1999; Morley, Goodwin, \& Darian-Smith, 1984; Nefs, Kappers, \& Koenderink, 2001; Van Boven \& Johnson, 1994; Van Doren, 1989). For example, Morley et al. investigated how well spatial period differences could be detected between two gratings of alternating grooves and ridges. They found that differences of about $5.6 \%$ could be detected for gratings with 2-mm spatial periods. Nefs et al. found spatial period discrimination thresholds ranging from $6.4 \%$ for sinusoidal gratings with $1.00-\mathrm{cm}$ spatial periods to $11.8 \%$ for sinusoidal gratings with $0.25-\mathrm{cm}$ spatial periods. In the present research, we investigated how well humans are able to detect differences in the line frequencies of two gratings. Line frequency is defined as the reciprocal of the shortest distance between the centers of two adjacent parallel lines. We also wanted to find out how well participants can detect gradual and periodic frequency changes within gratings.

This research was supported by Grant 575-24-001 from the Netherlands Organization of Scientific Research (NWO). We thank our participants for their valuable contributions. The authors are also indebted to Ronald Heijeler and Stefan Louw for constructing the UPS. Correspondence concerning this article should be addressed to H. T. Nefs, Department of Physics of Man, Universiteit Utrecht, Helmholtz Institute, Princetonplein 5, $3584 \mathrm{CC}$ Utrecht, The Netherlands (e-mail: h.t.nefs@phys.uu.nl).
Only Lamb (1983) has investigated the discrimination of dot density discrimination within a single texture by varying the dot density in horizontal and vertical directions in a dot matrix. He found that differences as little as $2 \%$ could reliably be detected. It is unfortunate that little research has been aimed at detecting change within textures, because the task of detecting change within a texture is quite different from detecting a difference between two separate textures. For example, since it is unclear how many line intervals are needed to perceive the line frequency in a line grating, we need to find out to what extent rapid variation and change of line frequency can be perceived or are masked within the texture. Masking of adjacent texture elements or patterns of texture elements that overlap in time is called lateral masking (Loomis \& Lederman, 1986). It is not an unreasonable thought that a gradual change in frequency is harder to detect than an abrupt change, since masking might prevent the detection of a difference in the distances spanned by two or more adjacent line intervals. Participants have to remember the spatial frequency over a longer distance and, therefore, over a longer period of time in order to overcome this problem. The detection of rapid periodic change requires that the size of the modulation period of the grating is larger than the minimum number of line intervals necessary to make an estimate of the line frequency; else, the modulation is missed.

Much research has been done to investigate the physical properties underlying roughness and the discrimination of roughness (e.g., Green, 1981; Green, Lederman, \& Stevens, 1979; Heller, 1982; Kudoh, 1988; Lederman, 1974, 1978, 1981, 1983). Heller, for example, reported that roughness 
discrimination could accurately be made on abrasives that differed by only $2-3 \mu \mathrm{m}$ in grid radius. Note that grid radius affects both height and grid density. The small differences found by Heller conform nicely to our own data on amplitude discrimination in sinusoidal gratings (Nefs et al., 2001). We found that amplitude differences as small as $2 \mu \mathrm{m}$ could reliably be detected between two sinusoidal gratings with a spatial period of $2.5 \mathrm{~mm}$.

Connor and Johnson (1992) investigated whether a roughness percept was caused by spatial variation on the skin or by variation in the firing rates of isolated neurons. They found that not so much the mean firing rate of neurons but the spatial variation in firing rate among neurons innervating the skin caused a roughness percept. Darian-Smith and Oke (1980) likewise showed that, on the periphery at least, spatial variation could be encoded only by populations of neurons, and not by individual neurons. Since the spacing in very fine textures is much smaller than the receptive fields of neurons, it is thought that temporal frequency is crucial for very fine texture discriminations (Connor \& Johnson, 1992). Vibrotactile research has shown that frequency discrimination thresholds increase from $20 \%$ to $30 \%$ of the standard frequency for frequencies ranging from 25 to $100 \mathrm{~Hz}$. Performance drops markedly for frequencies above $100 \mathrm{~Hz}$ (e.g., Goff, 1967; Rothenberg, Verrillo, Zahorian, Brachman, \& Bolanowski, 1977).

In all our experiments, the participants used active, dynamic touch. They felt the gratings with two fingersnamely, the index and middle fingers of the preferred hand-and moved them laterally across the gratings. Lateral motion has been found to be the natural exploratory procedure for extracting texture information from a surface (Lederman \& Klatzky, 1987). We agree with Gibson's $(1962,1966)$ view that active touch is preferable to passive touch, in which the stimulus is imposed upon the participant. The hand is not only a receptive organ, but also an exploratory device. Active touch might, therefore, be more sensitive to differences in spatial period. However, it also poses a problem, because participants might have to be able to accurately monitor their own hand speed in order to compare two successively felt gratings. The same holds for contact force. We felt that an important aspect of touch would be lost if we were to use the passive mode in our experiments. The participants were, therefore, free to determine their own speed of motion and their own preferred contact force, as long as hand speed was not so slow that they could count the individual lines.

In order to study discrimination thresholds for line frequency, we used gratings consisting of raised parallel lines on swell paper. ${ }^{1}$ In the first experiment, we determined the discrimination thresholds for line frequency when the participants compared two separate gratings successively. We wanted to determine the Weber fraction for line frequency discrimination and to find out whether it is constant in the measured range. In subsequent experiments, we studied the detection of frequency modu- lation within gratings. We distinguished two kinds of modulation: frequency transitions, as in gradient textures, and periodic change, as in sine-wave modulations. In the second experiment, we used line gratings with a frequency transition in the middle. The two sides of the texture had different frequencies. We measured the discrimination threshold for line frequency as a function of both frequency and the number of lines in the transition. In the third experiment, we periodically modulated the line frequency. That is, the distance between the lines was decreased and increased over fixed intervals. Detection thresholds for the modulation were determined for different base frequencies and for different sizes of the modulation period.

\section{EXPERIMENT 1 Frequency Discrimination}

What differences in spatial frequency can humans actually feel? And is the Weber fraction for line frequency discrimination constant with increasing frequency? These are very basic but unanswered questions. In this experiment, discrimination thresholds for line frequency were determined with the method of constant stimuli. The stimuli consisted of regular gratings of raised parallel lines. They were made of swell paper, which is easy to use and accurate enough for our purposes. In this range, the largest periods $(2 \mathrm{~cm})$ are larger than the width of a finger, and in the case of the smallest periods $(0.25 \mathrm{~cm})$, several periods fit on a single finger, which makes it an interesting range from an anatomical point of view. We expected that the Weber fraction might not be constant, since the participants might use different mechanisms for estimating the different line frequencies of the gratings in this range.

\section{Method}

Participants. Nine students at Utrecht University (5 women and 4 men; mean age, about 22 years) participated in this experiment. Five participants also participated in Experiment 3. All the participants were right-handed, according to the Coren (1993) test and had no callosities, scar tissue, or other signs of skin damage on their fingertips.

Stimuli. We printed parallel line gratings on rectangular sheets of swell paper, measuring $29.7 \times 10.5 \mathrm{~cm}$, with a 300 -dpi laser printer. Each line was $50 \mathrm{~mm}$ long, $0.8 \mathrm{~mm}$ thick, and $0.3 \mathrm{~mm}$ high. The total length of the gratings was $25 \mathrm{~cm}$. Heating the swell paper in order to raise the black lines did not alter the position of the lines; therefore, line patterns could be printed almost as accurately as on normal office paper. An example of a grating is shown in Figure 1.

We used four standard frequencies-namely, 0.5, 1, 2, and 4 lines $/ \mathrm{cm}$; these frequencies cover the entire range that can be made with this material. A grating with lower line frequencies would contain too few lines; higher frequencies were not possible because of the resolution of the swell paper. A standard grating was made for each frequency. Eight comparison gratings, with frequency steps ranging from $-21 \%(-1 \mathrm{~dB})$ to $+26 \%(+1 \mathrm{~dB})$ in 0.25 -dB intervals $^{2}(-21 \%,-16 \%,-11 \%,-6 \%, 6 \%, 12 \%, 19 \%$, and $26 \%)$ with respect to the standard, were made for each standard frequency. These gratings were used for 5 participants. Eight other comparison values, with frequency steps ranging from $-16 \%$ to $+19 \%(-16 \%$, 
Two fingers

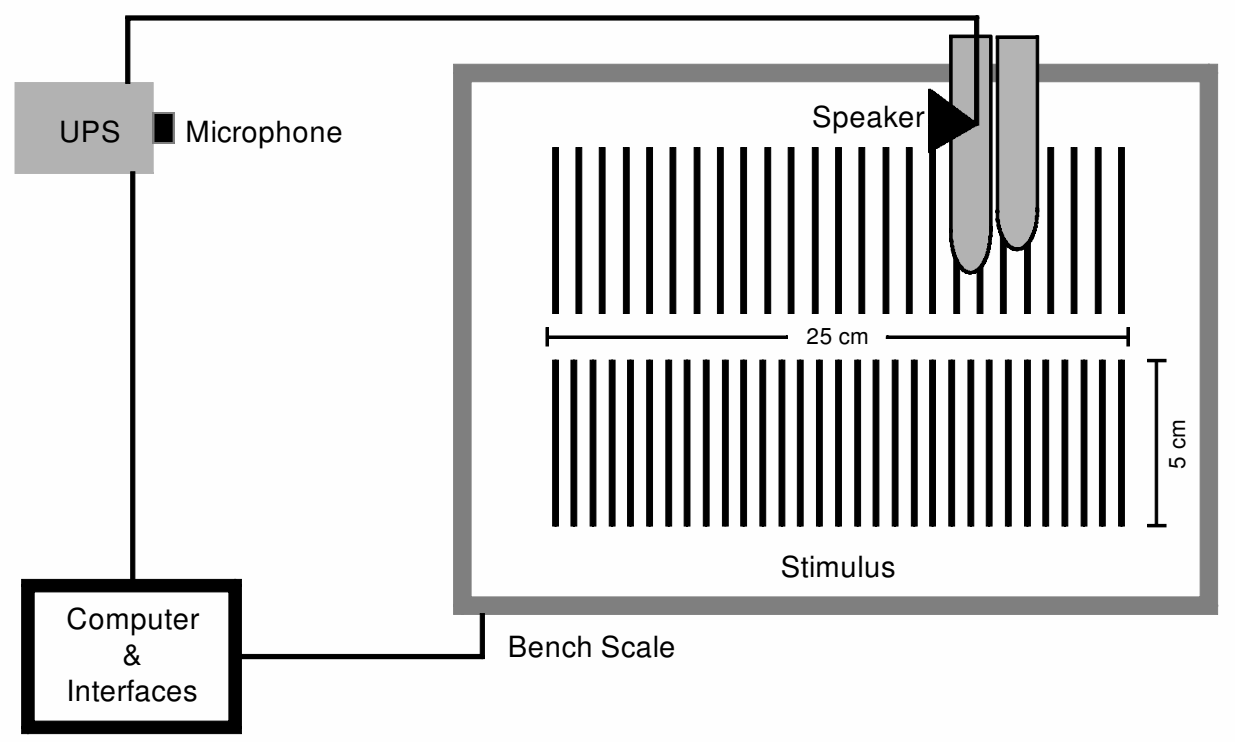

Figure 1. Display of the experimental setup of Experiment 1. The participants felt the grating with two fingers - that is, the index finger and the middle finger. The fingers were oriented parallel to the grating and moved perpendicularly across the grating. Two different gratings were printed on a single sheet of swell paper: One was a standard grating, and the other one was a comparison. After the participant had felt the first grating, the sheet was turned so that the second grating could be felt. In this figure the lower grating has the higher line frequency. The ultrasonic positioning sensor (UPS) consisted of a microphone and a speaker. The speaker was attached with a ring that was worn on the middle finger. The microphone was located at a distance of $80 \mathrm{~cm}$ from the edge of the stimulus. A bench scale was located beneath the stimulus. The top plate of the bench scale was level with the table, on which the elbow of the participant rested.

$-11 \%,-6 \%,-3 \%, 3 \%, 6 \%, 12 \%$, and $19 \%$ ) of the standard frequencies were used for the remaining 4 participants. We chose the comparison values on a logarithmic scale, since on this scale Weber fractions for increments and decrements are of the same size (provided the Weber fraction is constant). No comparison gratings were identical to the standard gratings. A comparison grating and a standard grating were always printed on the same sheet of swell paper (see Figure 1). Multiple sets were made so that each grating was felt only once in each experimental session. The number of times that a particular grating was touched was, therefore, so limited that replacing the gratings because of possible wear was not necessary.

Apparatus. An IBM-compatible, 100-MHz Pentium computer was used to handle all incoming measurements. Movement profiles were measured using an ultrasonic positioning sensor (UPS) operated by a modified Coach-Lab II $^{3}$ serial computer interface connected to the RS-232 port of the computer. The Coach-Lab II measured the time delay between the sending of a tone burst to a speaker and the reception of that signal at a microphone. This time delay was directly related to the distance between speaker and microphone. The participants had to wear a small speaker on a ring on their fingers. The microphone was located at a fixed position on the table at an $80-\mathrm{cm}$ distance from the edge of the stimulus. The UPS read in $1.7-\mathrm{cm}$ increments. Force measurements were made with a Mettler-Toledo "Spider-A6" bench-scale, which was connected to the second RS-232 port of the computer. Resolution was about $0.01 \mathrm{~N}$. The bench scale was lowered into the table so that the plate of the bench scale was level with the table. Figure 1 illustrates the experimental setup of this experiment. Force and position were sampled at $27 \mathrm{~Hz}$. This measurement frequency provided us with accurate and detailed movement and force profiles.
Procedure. The participants sat behind a curtain, which prevented them from seeing the stimuli, and they also wore earplugs to eliminate possible auditory cues. Instructions and procedural remarks, however, could still be heard. One hand was put under the curtain, while the elbow rested at a marked location on the table. The participant had to perform a two-alternatives forced-choice task. In each trial, we presented two gratings successively at the same place: a comparison and a standard grating, in random order. The participants had to decide which grating had the higher line frequency. We instructed them to move the index and middle fingers of the preferred hand (in each case, the right hand) twice from side to side and back again across the grating. The lines of the grating were perpendicular to the direction of motion and were oriented parallel to the finger. Speed and force measurements were obtained for 4 participants.

Every combination of comparison grating and standard grating was presented 16 times, which meant that each participant judged 512 trials (4 frequencies $\times 8$ frequency steps $\times 16$ presentations). We presented the trials in four or more sessions of, at most, 128 trials, taking approximately $1 \mathrm{~h}$ for each session. The experiment was divided into 16 blocks of 32 trials. In each block, only one standard frequency was used. The order between and within blocks was randomized, with the constraint that each standard frequency was used in each group of four consecutive blocks. Experiment 3 was run over the same period of time as Experiment 1. Sessions of Experiments 1 and 3 were held in random order.

The participants received written instructions, and these were also read aloud to them at the start of the first session. We repeated the instructions in an abbreviated form in the following sessions. The participants could ask additional questions if they wished. We 
started every block of 16 trials with 3 practice trials, to see whether the procedure was understood and to give the participant an opportunity to get used to the stimulus. Only the easiest comparisons were used as practice trials. We provided correctness feedback for every response during both the practice trials and the experimental trials. Force profiles and movement profiles were measured continuously during all the trials.

Analysis. Psychometric functions were fitted to the data for each standard frequency. Cumulative Gaussian ogives were used as psychometric functions. Two parameters, the mean and the standard deviation, could be fitted. Since the mean should be located, theoretically, at the standard value $(0 \%)$, only the standard deviation was fitted to the data; the mean was kept at the standard frequency. The discrimination threshold was defined as the frequency step that yielded the 0.75 score. These definitions are further illustrated in Figure 2.

\section{Results}

Threshold results. We determined the thresholds for four frequencies for all 9 participants. All the analyses were performed with decibels as units of measurements. Thresholds are reported in percentages of the reference frequency, which makes them easier to interpret. The calculated cumulative Gaussian ogives fitted the data well. Figure $3 \mathrm{~A}$ shows the average thresholds over the four standard frequencies for all the participants. The overall threshold was approximately $10.6 \%$ of the standard frequency. A considerable variation in the participants' mean thresholds was observed: $5.5 \%$ for participant D.A. and $16.1 \%$ for participant E.R. were the lowest and the highest mean thresholds, respectively.

The thresholds, averaged over all 9 participants, are plotted in Figure 3B as a function of line frequency. Thresholds, averaged over participants, decreased with the standard frequency, ranging from $13.0 \%$ for a standard frequency of 0.5 lines $/ \mathrm{cm}$ to $8.9 \%$ for 2 lines $/ \mathrm{cm}$. A one-way analysis of variance (ANOVA) with a repeated measures design indicated that there was a significant difference between the four frequencies $[F(3,24)=3.32$, $p=.04]$.
Speed and force results. Speed and force measurements are shown in Figure 4. Figures 4A and 4B are representative force and movement profiles of participant H.R. as a function of time. The average force is obtained by dividing the sum of all force measurements in a trial by the number of measurements. The average speed in a trial is obtained by dividing the sum of all differences in position on consecutive measurements by the number of measurements minus one. Average force and average speed are shown for all the trials of participant H.R. in Figures 4C and 4D, respectively. The average speed was about $0.26 \mathrm{~m} / \mathrm{sec}$ and ranged from $0.12 \mathrm{~m} / \mathrm{sec}$ for participant H.H. to $0.44 \mathrm{~m} / \mathrm{sec}$ for L.W. As can be seen in Figure 4D, the speed used was also dependent on the trial number. This was typical for all the participants. Slopes of the bestfitting linear functions ranged from $6.7 * 10^{-4} \mathrm{~m} /(\mathrm{sec}$ trialnr) for participant E.R. to $1.7 * 10^{-4} \mathrm{~m} /(\mathrm{sec}$ trialnr) for participant H.H. This amounts to an increase of $0.345 \mathrm{~m} / \mathrm{sec}$ in scanning speed for E.R. and $0.085 \mathrm{~m} / \mathrm{sec}$ for H.H. over the entire experiment. The average force for a participant was about $1.37 \mathrm{~N}$ and ranged from $0.62 \mathrm{~N}$ for participant H.H. to $2.76 \mathrm{~N}$ for H.R.

We calculated the mean force for each standard frequency. These values are presented in Figure 4E. Standard deviations are corrected for the linear increase with trial number. Applied force increased with increasing standard frequency from $1.24 \mathrm{~N}$ for gratings with 0.5 line $/ \mathrm{cm}$ to $2.07 \mathrm{~N}$ for the gratings with 4 lines $/ \mathrm{cm}$. We tested this with a 256 trials $(16$ repetitions $\times 8$ comparisons $\times 2$ first/second) $\times 4$ participant $\times 4$ frequency ANOVA. The effects of frequency and participant and the interaction between them were all significant $[F(3,4005)=$ $615.76, p<.001, F(3,4005)=3764.26, p<.001$, and $F(9,4005)=83.03, p<.001$, respectively]. We also tested this with the polynomial contrasts and found that the linear trend was significant $[F(1,4005)=1798.47$, $p<.001]$. We also tested whether the observed trends were significant for individual participants and found

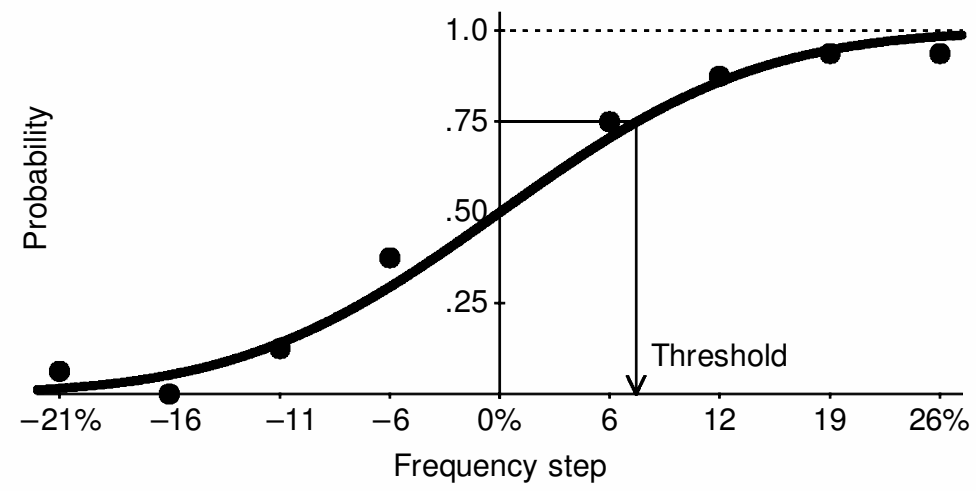

Figure 2. The psychometric curve. The probability of the participant's responding that the comparison stimulus has a higher line frequency than the standard grating is plotted as a function of the frequency step of the comparison gratings. 

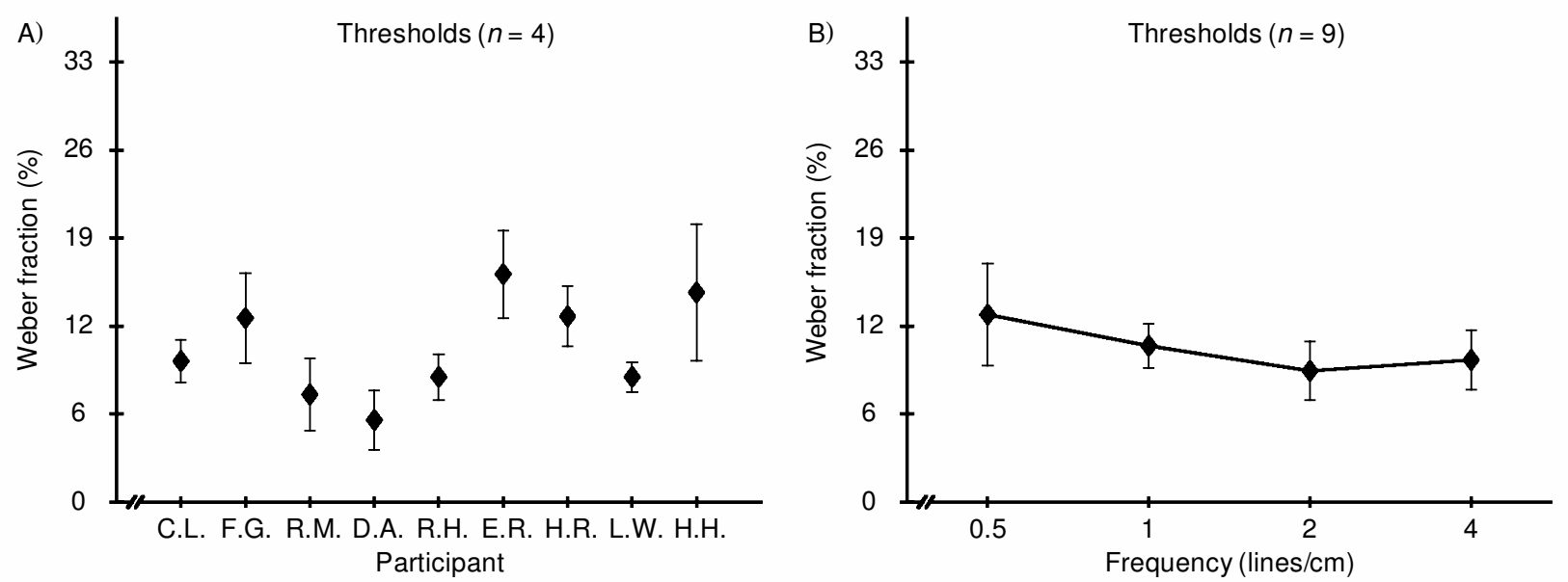

Figure 3. Threshold results of Experiment 1. (A) Average results for all the participants. Standard deviations are corrected for between-frequency differences. (B) Average thresholds for the four standard frequencies. Standard deviations are corrected for the between-subjects differences.

that the linear trends were significant for all 4 participants $[F(1,997)=152.86, p<.001, F(1,1004)=561.73$, $p<.001, F(1,1012)=879.34, p<.001$, and $F(1,992)=$ $398.45, p<.001$, for E.R., H.R., L.W., and H.H. respectively]. The linear trends were positive for all the participants.

We also calculated the mean speed for each standard frequency. These values are presented in Figure 4F. Hand speed decreased with increasing standard line frequency from $0.27 \mathrm{~m} / \mathrm{sec}$ for 0.5 line $/ \mathrm{cm}$ to $0.25 \mathrm{~m} / \mathrm{sec}$ for 4 lines $/ \mathrm{cm}$. Standard deviations are corrected for the linear increase with trial number. We tested this with a 256 trials (16 repetitions $\times 8$ comparisons $\times 2$ first $/$ second $) \times 4$ participant $\times 4$ frequency ANOVA. We found that different speeds were used for different frequencies $[F(3,4005)=$ $20.79, p<.001]$. There was also a significant difference between participants $[F(3,4005)=4506.26, p<.001]$ and a significant interaction between participant and frequency $[F(9,4005)=41.46, p<.001]$.

\section{Discussion}

We found that the lower line frequencies were harder to discriminate than the higher line frequencies. We also found that the applied force increased with line frequency. A probable explanation is that participants can increase the amplitude of indention on their skin when the skin has not reached the bottom of the grooves between the lines yet. In other words, they can still heighten the contrast on their fingers between the skin that touches the ridges and the skin that does not touch the stimulus. In the case of the lower line frequencies, an entire finger can fit between the lines. Pressing harder does not improve the contrast on the finger. In the case of the higher line frequencies, several lines fit on a single finger; if the lines are close together, one has to press harder to reach the bottom of the grooves.

We found significant effects of frequency on the scanning speed. Note, however, that although these effects were highly significant, they were relatively small (not more than $25 \%$ of the hand speeds), as compared with the eightfold increase in temporal frequency on the skin. This might suggest that participants do not try to keep the temporal frequency constant over different line frequencies when feeling the gratings and that they might have a way to get a sensation from the stimuli that is invariant over scanning speed.

\section{EXPERIMENT 2 Grating Segregation}

How well are humans able to detect a frequency modulation within a single grating? We addressed this question in Experiments 2 and 3. In Experiment 2, we determined the thresholds for grating segregation for two standard frequencies (1 and 2 lines/cm). Line gratings with two different halves were used: one with the standard frequency and the other with a comparison frequency that could be higher or lower than the standard. The participants were asked to indicate the direction of the transition. The same questions were raised as those in Experiment 1. In addition, we wanted to find out whether grating segregation is easier if the change is abrupt than if it is gradual. A priori, it is not evident what effect the length of the transition has on haptic grating segregation. For this reason, we manipulated the transition length by altering the number of line intervals over which the frequency changed.

\section{Method}

Participants. Five students at Utrecht University (4 men and 1 woman; mean age, about 19 years) participated in this experiment. None of them participated in Experiments 1 and 3. The students had no callosities, scar tissue, or other signs of skin damage on their fingertips. Participants L.S. and I.V. were left-handed, and the others were right-handed, according to Coren's (1993) questionnaire. They were paid for their contribution.

Materials. Gratings were printed on a rectangular sheet of swell paper $(29.7 \times 10.5 \mathrm{~cm})$ and with the same specifications as those in 

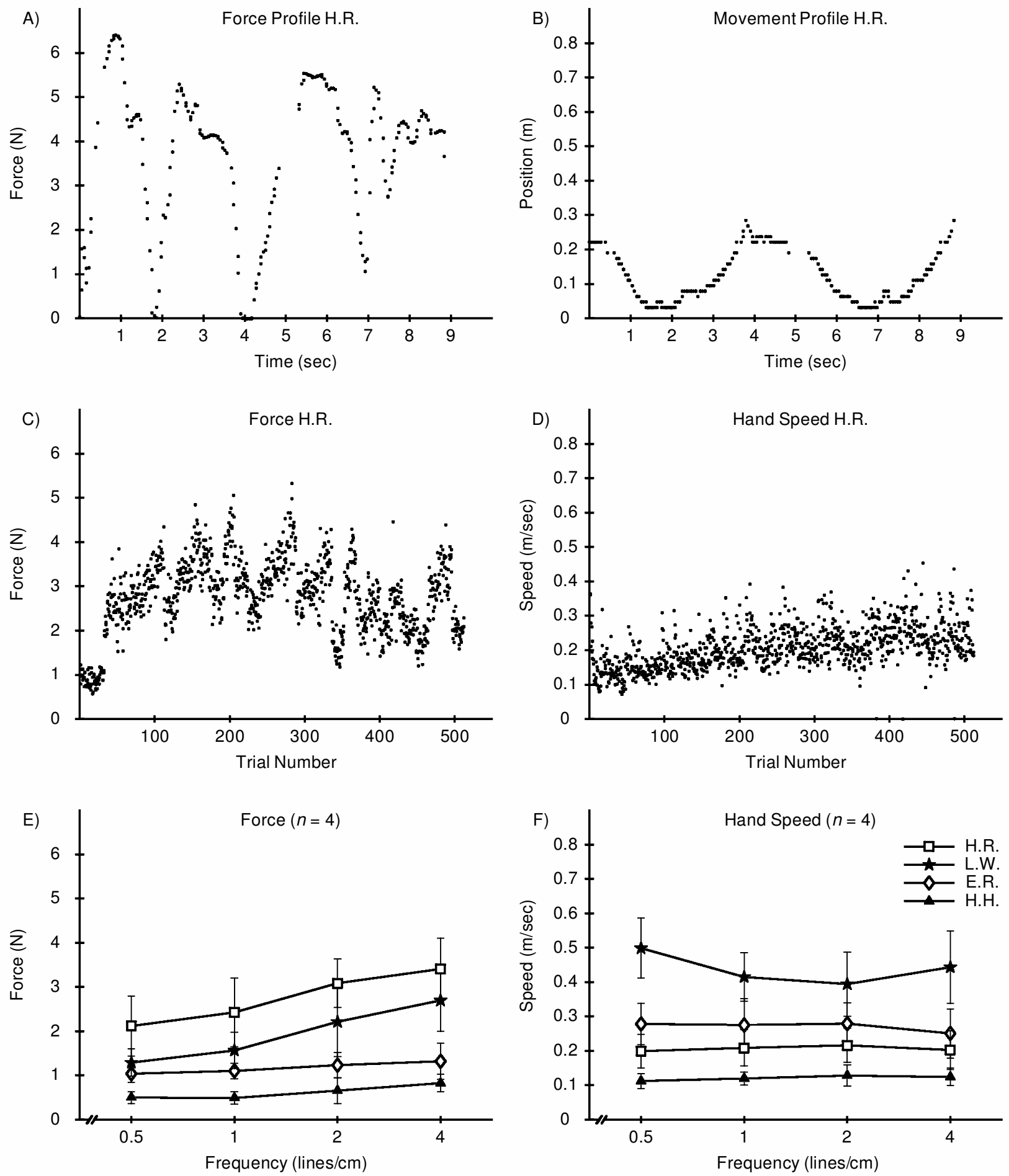

Figure 4. Force and speed results of Experiment 1. (A) Example of the force profile of a single movement of H.R. (B) Example of the movement profile of a single movement of H.R. (C) Average force of a movement profile as a function of trial number. (D) Average speed of a movement profile as a function of trial number. (E) Average force as a function of standard frequency for 4 participants. (F) Average speed as a function of standard frequency for 4 participants.

Experiment 1. One side had a standard frequency of 1 or 2 lines $/ \mathrm{cm}$, and the other side had a frequency step ranging from $-21 \%$ $(-1 \mathrm{~dB})$ to $+26 \%(+1 \mathrm{~dB})$ of the standard frequency in $0.25 \mathrm{~dB}$ steps $(-21 \%,-16 \%,-11 \%,-6 \%, 6 \%, 12 \%, 19 \%, 26 \%)$. In no case was the line frequency the same on both sides of the grating. The number of line intervals needed for the transition between the standard and the comparison frequency was also manipulated $(0$, 16, and 32 line intervals). The last condition (32 line intervals) was 
not used for the 1-lines/cm condition, since in that case, the frequency transition exceeded the grating boundaries. The sizes of the line intervals - that is, the center-to-center spacings between two adjacent lines - in the transition were calculated according to Equation 1:

$$
\text { interval width }(n)=\frac{1}{f_{\mathrm{r}}}\left(\frac{f_{\mathrm{r}}}{f_{\mathrm{t}}}\right)^{\frac{n}{M+1}} \text {. }
$$

The interval size was a constant percentage of the previous one. $M$ is the total number of intervals in the transition, $n$ is the number of the current interval, and $f_{\mathrm{r}}$ and $f_{\mathrm{t}}$ are the standard and comparison frequencies, respectively. The middle position of the transition was situated at the center of the grating. Examples of the stimuli are given in Figure 5.

Procedure. The same experimental setup was used as that in Experiment 1 . However, this time, the participants felt only one stimulus in each trial. They had to indicate which side of the grating had the higher line frequency. All the stimuli were presented 16 times, which amounted to 640 trials ( 5 conditions $\times 8$ frequency steps $\times$ 16 presentations); about $5 \mathrm{~h}$ were required for each participant. The trials were given in six sessions of six or seven blocks, each block consisting of 16 trials. Only one condition was tested in each block. Each condition was tested once or twice at each session. The order of blocks and of trials was randomized. Instructions and practice trials were as in Experiment 1. Correctness feedback was provided for both practice trials and experimental trials.

Participants I.V. and J.N. did an additional session. After six sessions, participant I.V. repeated the first one, because her accuracy in the first session was much lower than in the other five. The first one was replaced by the seventh session. Participant J.N. repeated one condition (1 line/cm, with a transition length of 16 line intervals) in an extra session because a psychometric function could not be fitted to the data for that condition.

Analysis. The same method of analysis was used as that in Experiment 1 .

\section{Results}

Threshold results for all 5 participants and the average threshold results are presented in Figure 6. All the analyses were again performed with decibels as units of measurements. Average thresholds for the six conditions ranged from $8.6 \%$ to $10.9 \%$. The participants did not seem to be influenced by the number of line intervals in the transition. As can already be seen in Figure 6, the participants may have found one frequency more difficult than another frequency. The frequency level that yielded the lower thresholds, however, was not consistent across participants.

Since the design was nonorthogonal, we analyzed the thresholds in two separate designs. We performed repeated measures ANOVAs on both designs. The first design involved $2 \times 2$ frequency $(1$ and 2 lines $/ \mathrm{cm}) \times$ transition (0 and 16 line intervals). Neither the effect of

\section{Transition length (lines)}

0

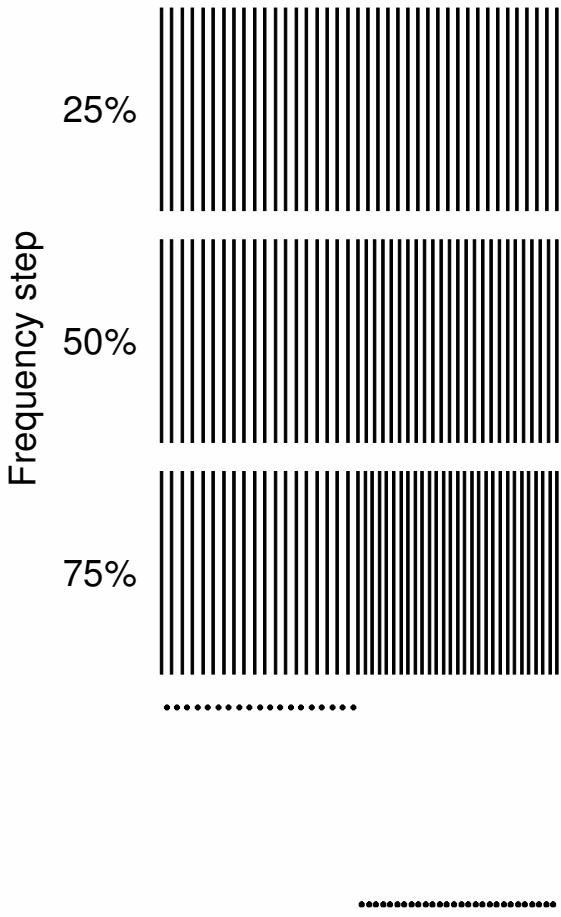

16
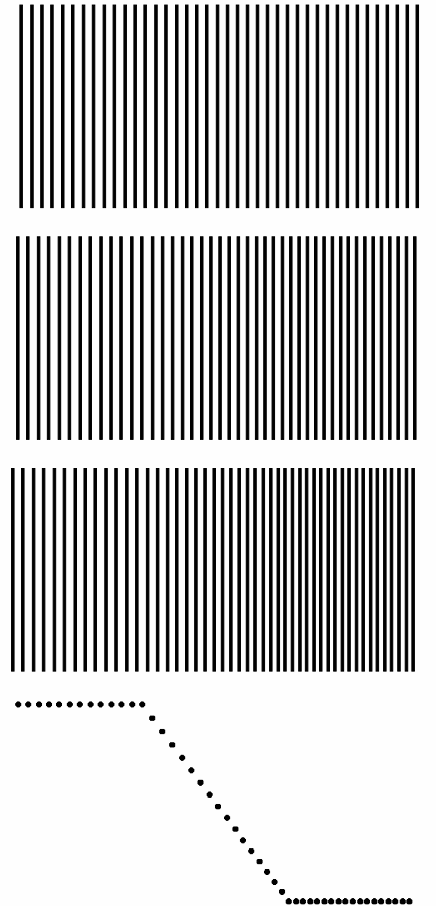

32
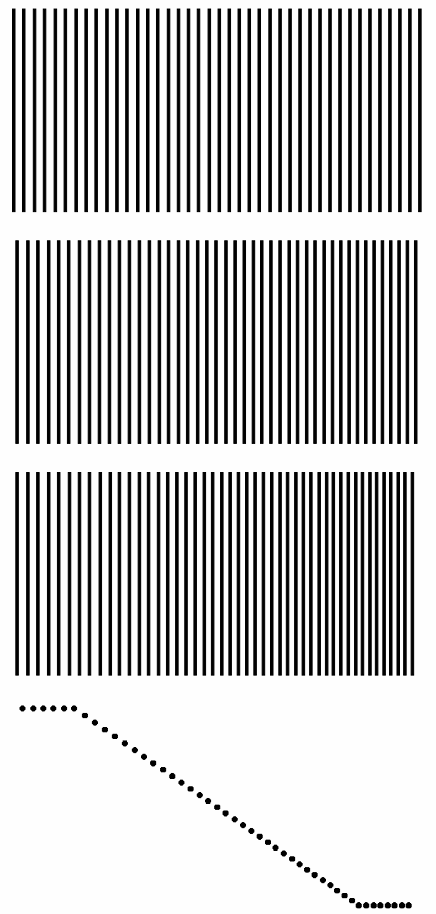

Figure 5. Examples of the stimuli in Experiment 2. One side of the grating had the standard line frequency, and the other side had a comparison frequency. In these examples, the comparison frequency is always on the right side of the stimulus. The comparison frequency can be higher or lower than the standard frequency. Only higher comparison frequencies are plotted. The widths of the line intervals in the bottom gratings are plotted schematically in the dot diagrams as a function of the position of the center of the line interval. 

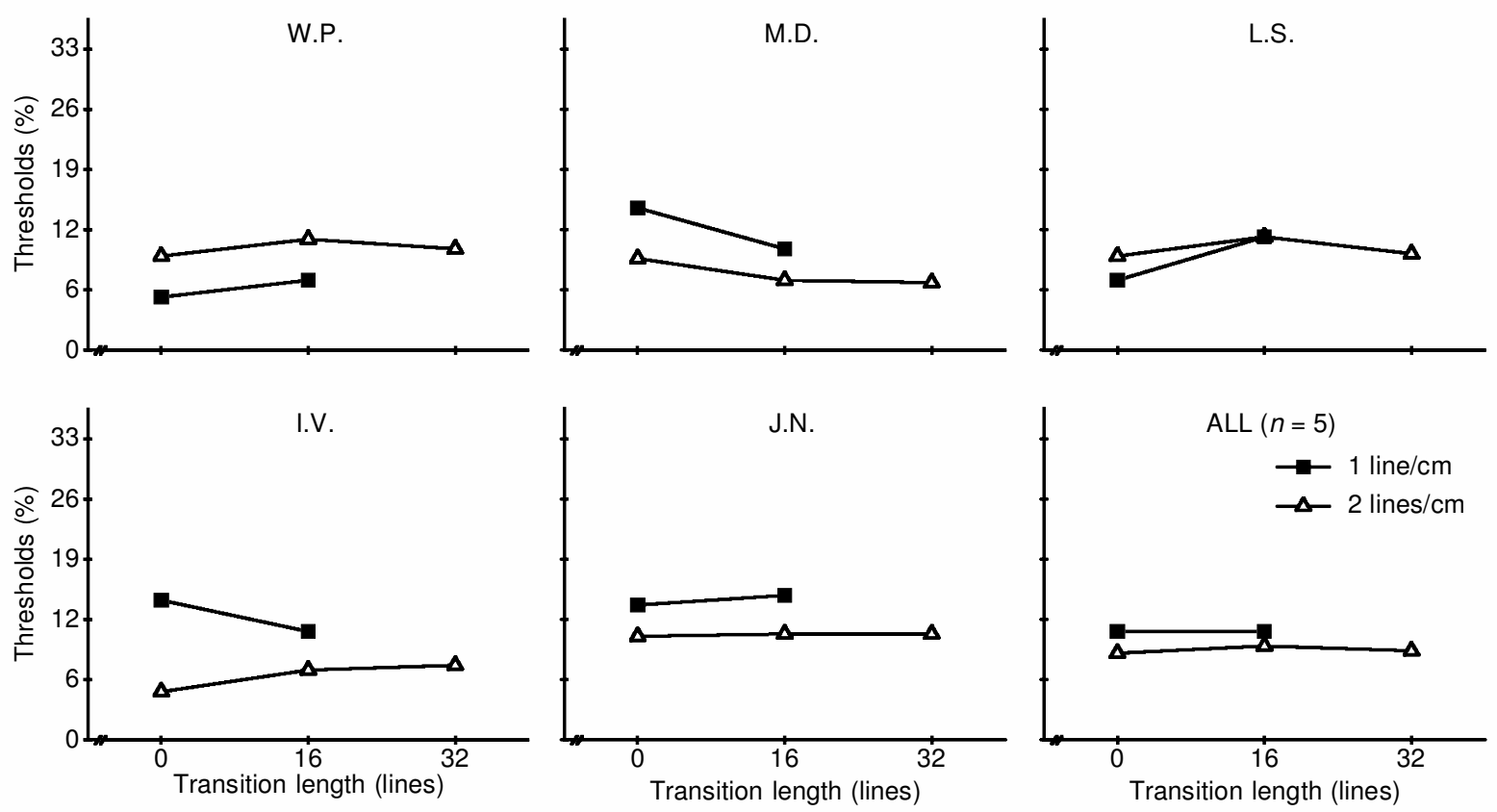

Figure 6. Results of Experiment 2. Discrimination thresholds are plotted as a function of the number of line intervals in the transition for each participant. The two lines represent the two frequency levels. The bottom right figure shows the average results over all participants.

frequency or transition nor the interaction was significant $[F(1,4)=0.84, p=.41, F(1,4)=0.12, p=.75$, and $F(1,4)=0.39, p=.57$, respectively $]$.

The second design was a $1 \times 3$ frequency $(2$ lines $/ \mathrm{cm})$ $\times$ transition $(0,16$, and 32 line intervals) design. There was no significant main effect of transition $[F(2,8)=$ $0.63, p=.56]$ in this design either. In short, no significant effects were found.

\section{Discussion}

Thresholds were similar to those found in Experiment 1 . Apparently, it does not matter whether the gratings are separate or joined together. A visual inspection of the examples given in Figure 5 seems to indicate that gradual change might be more difficult to perceive than abrupt changes. Cornsweet (1970, pp. 268-364) also has suggested that, because of low-frequency attenuation in the visual system, a gradual change in illuminance might be more difficult to perceive than a sharp transition. Such a dependency of thresholds on transition length, however, is not found in the tactual modality.

Another way to consider the stimulus is not by the length of the transition, but by the size of the constant area. The stimuli with a large transition length have a small area where the frequency is constantly high or low. This constant area is of limited extent, and in some conditions, it is even rather small, as compared with the transition. One can also conclude from this that the minimum area of constant frequency necessary to compare it with a second area may be very small, or that the constant area is perhaps not used at all.

\section{EXPERIMENT 3 Detection of Periodic Change}

The second kind of frequency modulation that we used was periodic change. In Experiment 3, we studied the detection thresholds for periodic frequency modulation in line gratings. The participants always compared a standard grating with a constant line frequency and a comparison grating that was periodically modulated. This was a comparison between two gratings. However, in order to detect the difference between the two gratings, the participants had to detect the modulation. The frequency modulation was the only parameter for discriminating between the two gratings. It should be emphasized that the average line frequency was equal for the two gratings. For this reason, we consider this to be a true discrimination task for line frequencies in a single grating.

To detect the modulation, the participant had to detect the difference between at least two different line frequencies - that is, the highest and the lowest line frequencies. However, it has not yet been established that a single line interval in a grating is sufficient to extract the line frequency at that point in the grating. More line intervals might be required. The modulation cannot be detected if the modulation period is smaller than the minimum area or the number of line intervals needed.

In this experiment, we varied both the base frequency and the size of the modulation period, and for these conditions, we determined the minimum modulation depththat is, the amount or strength of contraction and expansion-needed to detect the modulation. 


\section{Method}

Participants. Five students who participated in Experiment 1 also took part in this experiment.

Materials. The stimuli were made of swell paper with the same specifications as those in Experiments 1 and 2. In this experiment, two kinds of gratings were used. First, we used gratings with a constant line frequency of 1 or 2 lines $/ \mathrm{cm}$, which we called the standard gratings. Second, comparison gratings were constructed by periodically modulating the frequency of the standard gratings. The size of the modulation period was two, four, or eight lines. If larger modulation periods were used, only one or two periods would fit in the grating. In that case, the gratings would start to resemble the gratings used in Experiment 2. One standard grating and six comparison gratings were used for each combination of frequency and modulation period. The comparison gratings varied from $4 \%$ to $39 \%$ modulation depth in equilog distances (approximately $4 \%-6 \%-$ $10 \%-16 \%-25 \%-39 \%)$. Standard gratings, of course, had $0 \%$ modulation depth. The position of each line in the grating was calculated from Equation 2:

$$
\text { position }(n)=\frac{n}{f_{\mathrm{c}}}+\frac{M}{2 \pi \cdot f_{\mathrm{m}}} \cos \left(\frac{2 \pi \cdot f_{\mathrm{m}} \cdot n}{f_{\mathrm{c}}}\right) .
$$

In this equation, $n$ is the serial number of the parallel line in the grating, $f_{\mathrm{c}}$ is the carrier or base frequency, and $f_{\mathrm{m}}$ is the modulation frequency. $M$ is the modulation depth-that is, the amount/strength of contraction and expansion. The number of lines in a modulation period is given by the ratio of the carrier frequency to the modulation frequency. $M$ has to be multiplied by 0.64 for the 2 - and 4-lines/modulation-period conditions and by 0.90 for the 8 -lines/ modulation-interval conditions to get the peak-to-baseline difference ratio in line frequency in a modulated grating. Figure 7 shows some examples of the stimuli.

Procedure. The experimental setup was the same as those in Experiments 1 and 2 . Two gratings were presented to the participant successively and in random order. One was a regular grating, and the second was modulated periodically. The participant had to say whether the first or the second grating was modulated.
All the grating pairs were presented 32 times. This amounted to 1,152 trials ( 2 frequencies $\times 3$ modulation periods $\times 6$ modulation depths $\times 32$ presentations) for each participant. The trials were presented in 12 sessions, each consisting of 96 trials. The order of the sessions was randomized. Only one condition was tested in each session. A session consisted of 16 trials with all six modulation depths of one experimental condition. The participants needed about $3 / 4 \mathrm{~h}$ to complete a single session. The order of the trials within a session was randomized. Every session started with an instruction and five practice trials involving the easiest comparisons. We provided correctness feedback for our participants during both practice trials and experimental trials.

Analysis. Cumulative Gaussian functions were fitted as in Experiment 1 . The probability of correctly identifying the comparison grating is a function of the modulation depth. The asymptotic values of the function were now at chance level $(50 \%)$ and at the $100 \%$ correct score. Both the mean and the standard deviation were fitted to the data. The mean was the value that yielded the $75 \%$ score and, in this case, represented the threshold. The slope of the curve at the mean was inversely related to the standard deviation.

\section{Results}

Two thresholds were out of range for participant F.G. She performed at chance level in these conditions at all the modulation depths used. Thresholds could, therefore, not be adequately measured. We have excluded the results for this participant from the statistical analysis. Thresholds are plotted in Figure 8 as a function of the modulation period for all 5 participants. The average thresholds varied between $13.5 \%$ and $19.9 \%$ for the six experimental conditions. As can be seen, for most of the participants, the thresholds for the 2-lines/cm condition were lower than those for the 1-line/cm condition. A $2 \times$ 3 frequency ( 1 and 2 lines/cm) modulation period (2, 4, and 8 lines) ANOVA with a repeated measures design,

\section{Modulation period (lines)}

2

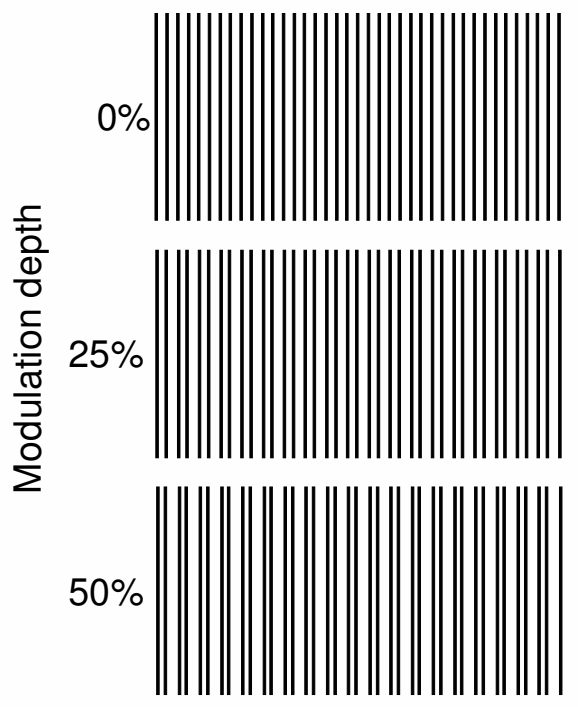

4
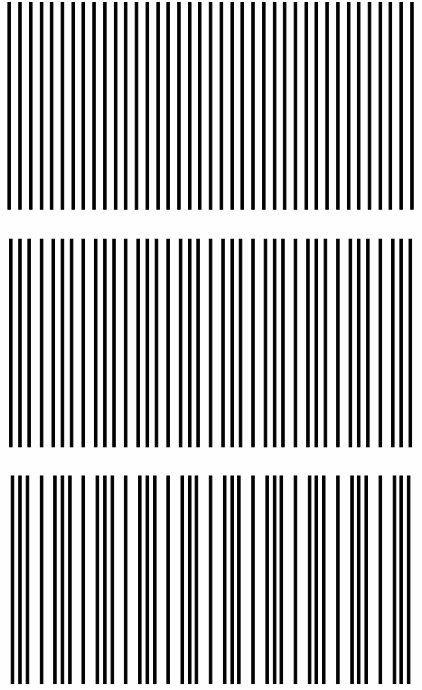

8
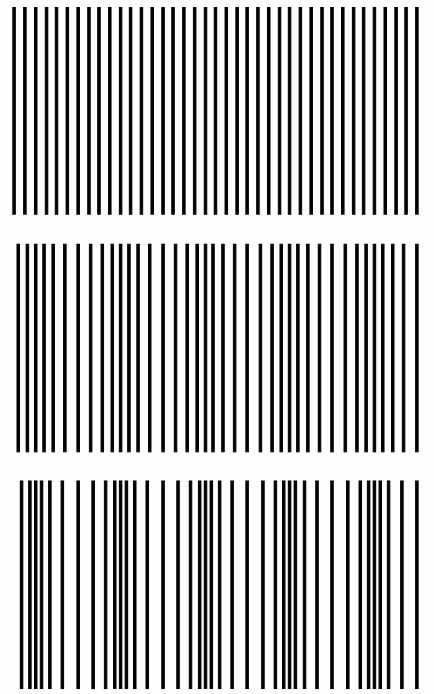

Figure 7. Examples of the stimuli in Experiment 3. The upper gratings represent the standard gratings. The lower gratings are modulated periodically. The lowest gratings have the largest modulation depth. 

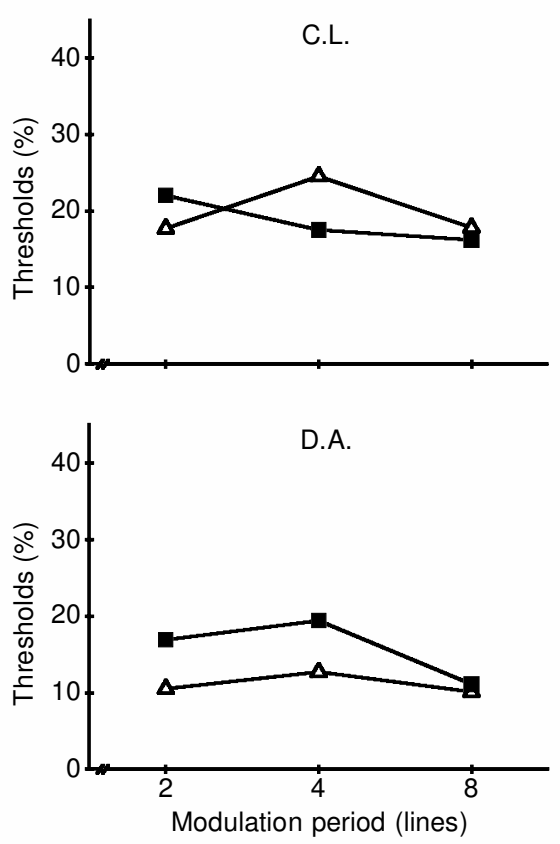
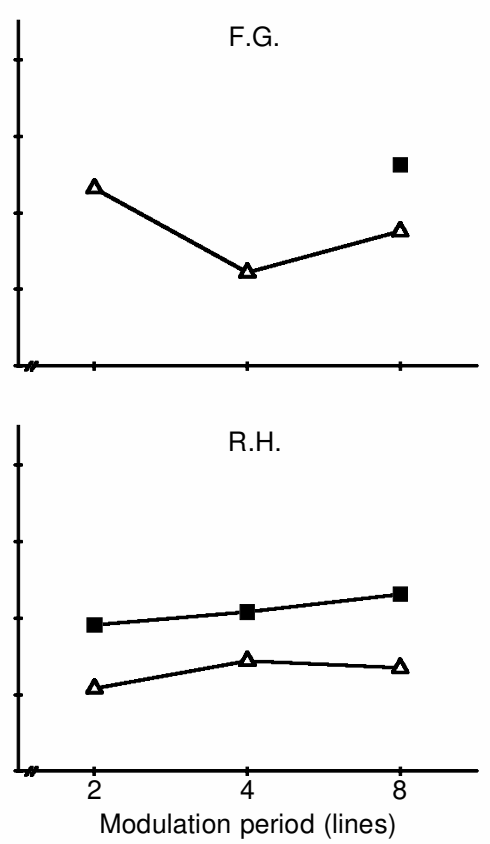

R.M.
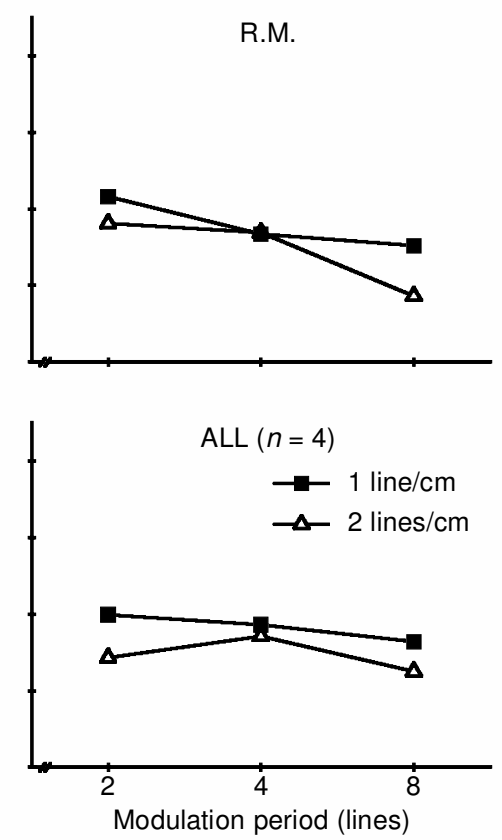

Figure 8. Results of Experiment 3. Thresholds are plotted as a function of the number of lines in a modulation period. The two lines represent the two frequency levels. The bottom right figure shows the average results for all participants except participant F.G., because of missing data.

however, showed no significant main effect for frequency $[F(1,3)=3.44, p=.16]$. Note that F.G.'s results suggested an effect of frequency, as did the results of the other participants. As would already be expected from Figure 8 , there was no significant effect of modulation period $[F(2,6)=2.12, p=.20]$. The interaction between frequency and modulation period was not significant either $[F(2,6)=1.34, p=.33]$.

\section{Discussion}

Apparently, the size of the modulation period was of little importance when expressed as the number of lines per modulation interval or when expressed in centimeters, since there was no interaction between modulation period and frequency. Although the data suggested an effect of frequency-namely, that the thresholds for the 2-lines/cm condition were lower-this effect was found to be nonsignificant. Apparently, the participants could detect the rapid modulation just as accurately as they could the modulation in long modulation periods. Thus, effects of lateral masking were not observed in this task.

\section{GENERAL DISCUSSION}

The major finding in Experiment 1 was that the Weber fraction for discriminating between line frequencies of separate gratings decreased within the experimental range. The results of Experiment 2 showed that frequency had no significant effect on the thresholds in the grating segregation task; the thresholds were similar to those found in Experiment 1. Segregating gratings is, in that respect, similar to discriminating between two separate gratings. The analysis in Experiment 3 also yielded no significant effect of base frequency, although the results suggested that, for most of the participants, the 2-lines/cm condition was easier than the 1-line/cm condition.

Thresholds in Experiments 1 and 2 were larger than those in the studies of Morley et al. (1984) and Nefs et al. (2001). Apparently, the line gratings were perceptually different from the sinusoidal gratings used by Nefs et al. or the gratings used by Morley et al. Of course, especially the 0.5 -line/cm line gratings were quite different from sinusoidal gratings. The 4-lines/cm line gratings were much more like sinusoidal gratings, because the ridges and the grooves were about the same size. Note that in that case, the discrimination threshold in the present study $(8.8 \%)$ was more similar to the discrimination threshold (11.8\%) found by Nefs et al. An important difference with Morley et al.'s study was that they used highly trained participants. It is possible that this caused the differences in thresholds.

Another remarkable finding was that the changes in thresholds as a function of standard frequency were surprisingly small from a physiological point of view, since the frequency range is quite large. In the case of Experiment 1 , there was even an eightfold increase in line frequency. In the lowest line frequency conditions, only one line was at the fingertip at the same time, whereas in the higher line frequency conditions, several lines were at the fingertip simultaneously. If the line frequency of a grating is perceived from the spatial pattern it imposes 
on the skin, one should have expected this to have had a substantial influence on the thresholds, since the size of the spatial imprint was limited by the size of the finger.

The eightfold increase is, of course, present not only in the spatial domain, but also in the temporal domain. If spatial layout is discarded, each point on the skin can be considered as an independent measuring device of temporal frequency. In principle, it is possible to discriminate between gratings by temporal frequency alone. However, if temporal information is used in comparing two gratings, the speed of the hand has to be taken into account. In other words, the sensation one wants to use for comparing the gratings has to be speed invariant. We showed that our participants changed their scanning speed only a little across different conditions; the change in scanning speed was about 32 times $(800 \% / 25 \%)$ smaller than the increase in line frequency. Nevertheless, there was considerable variation between and within trials. Apparently, the participants did not need to adjust their speed much in order to get a speed-invariant sensation.

It has been found in vibrotactile research that the Weber fraction for pulse rate in tactually applied vibration on the forearm is fairly constant-namely, $10 \%$-in the range from 10 to $50 \mathrm{~Hz}$. The Weber fraction rises to about $35 \%$ if the pulse rate is increased to $300 \mathrm{~Hz}$ (Rothenberg et al., 1977). Goff (1967) also found that the discrimination threshold was constant at about $10 \%$ up to $100 \mathrm{~Hz}$ and increased after that. It is interesting that we found a trend toward decreasing thresholds as a function of an increase in line frequency, whereas Goff and Rothenberg et al. found trends toward increasing thresholds for vibrotactile discrimination. In Experiment 1, we found that scanning speed was nearly constant. Therefore, the temporal frequency increased with increasing line frequency. If we calculate the temporal frequencies on the skin for each line frequency, we see that they range from about 13 to $100 \mathrm{~Hz}$. For example, with a mean hand speed of $0.26 \mathrm{~m} / \mathrm{sec}$ and a line frequency of 4 lines $/ \mathrm{cm}$, the temporal frequency is 104 lines $/ \mathrm{sec}$ $(0.26 * 400)$. Individual participants go far outside this range, but this has no obvious effect on the thresholds. Of course, for each participant, there were periods in a trial that the fingers moved at a speed that was lower than the mean speed-for example, at the end of a grating.

A limitation of the studies by Rothenberg et al. (1977) and Goff (1967) is that they used single vibrator points. We think that it is incorrect to consider temporal discrimination to be independent of spatial characteristics when studying the discrimination of gratings. For example, it is unclear whether temporal frequency should be considered to be the number-of-pulses/sec at a certain point or line at the skin or the summed number-of-pulses/sec in an area on the skin. It is also unclear whether participants are able to estimate the hand speed relative to the surface from the changes on the skin itself or use an efference copy, or kinesthetic and proprioceptic sensations instead. Darian-Smith, Davidson, and Johnson (1980) proposed that the direction and speed of motion is retained in the population of neurons innervating the fingerpad. Van Doren (1989) used traveling and static sinusoidal waves with a multicontactor array, but he did not address this possibility.

In Experiment 2, we found that the number of lines in the transition does not affect a participant's ability to discriminate between two line frequencies. Apparently, our participants were able to retain an accurate estimate of the line frequency on one side of the grating when crossing the gradient part of the grating. In other words, the percept was not contaminated by the line frequency transition.

The size of the modulation period in Experiment 3 had no effect on the thresholds either. Apparently, the participants were able to follow the rapid change in line frequency in the small modulation periods, as well as the change in larger modulation periods. We did not find any indication that frequency modulation is masked because of a limited temporal or spatial resolution. That is, lateral masking, if present at all, is not related to the size of the modulation period in periodic variation or to the base frequency in this range. However, this does not mean that lateral masking does not occur in gratings. We note that in Experiment 3, the peak-to-baseline difference ratio in line frequency owing to the modulation depth at detection threshold was larger than the discrimination thresholds found in Experiments 1 and 2. Comparison of the results of Experiments 1 and 2 with those of Experiment 3 is difficult, since the tasks were quite different.

Although we did not measure scanning speed, as in Experiment 1, we are certain that the participants did not alter their scanning speed much across the two different frequency conditions. This implies that temporal frequency and spatial frequency are linked; that is, changes in spatial frequency result in equivalent changes in temporal frequency, and vice versa. It might also be concluded that the participants did not try to alter the temporal frequency by changing their scanning speed, in order to perceive the gratings at the temporal frequency at which they were most sensitive to variation in temporal frequency.

In summary, we conclude three things. First, the Weber fraction is not constant for line frequency discrimination. Second, it does not matter if the transition length between two line frequencies extends beyond the width of the finger. Finally, modulation detection thresholds do not depend on the spatial frequency of the modulation.

\section{REFERENCES}

Connor, C. E., \& Johnson, K. O. (1992). Neural coding of tactile texture: Comparison of spatial and temporal mechanisms for roughness perception. Journal of Neuroscience, 12, 3414-3426.

Coren, S. (1993). The left hander syndrome. New York: Vintage Books. Cornsweet, T. N. (1970). Visual perception. London: Academic Press.

DARIAn-Smith, I., Davidson, I., \& Johnson, K. O. (1980). Peripheral neural representation of spatial dimensions of a textured surface moving across the monkey's finger pad. Journal of Physiology, 309, 135-146. 
Darian-Smith, I., \& OKe, L. E. (1980). Peripheral neural representation of the spatial frequency of a grating moving across the monkey's finger pad. Journal of Physiology, 309, 117-133.

Gibson, J. J. (1962). Observations on active touch. Psychological Review, 69, 477-491.

Gibson, J. J. (1966). The senses considered as perceptual systems. Boston: Houghton Mifflin.

GoFf, G. D. (1967). Differential discrimination of frequency of cutaneous mechanical vibration. Journal of Experimental Psychology, 74, 294-299.

Green, B. G. (1981). Tactile roughness and the "paper effect." Bulletin of the Psychonomic Society, 18, 155-158.

Green, B. G., Lederman, S. J., \& Stevens, J. C. (1979). The effect of skin temperature on the perception of roughness. Sensory Processes, 3, 327-333.

Heller, M. A. (1982). Visual and tactual texture perception: Intersensory cooperation. Perception \& Psychophysics, 31, 339-344.

KuDOH, N. (1988). Tactile perception of textured surfaces: Effects of temporal frequency on perceived roughness by passive touch. Tohoku Psychologica Folia, 47, 21-28.

Lamb, G. D. (1983). Tactile discrimination of textured surfaces: Psychophysical performance measurements in humans. Journal of Physiology, 338, 551-565.

Lederman, S. J. (1974). Tactile roughness of grooved surfaces: The touching process and effects of macro- and microsurface structure. Perception \& Psychophysics, 16, 385-395.

Lederman, S. J. (1978). Heightening tactile impressions of surface texture. In G. Gordon(Ed.), Active touch: The mechanism of recognition of objects by manipulation. A multi-disciplinary approach (pp. 205214). Oxford: Pergamon.

Lederman, S. J. (1981). The perception of surface roughness by active and passive touch. Bulletin of the Psychonomic Society, 18, 253-255.

LEDERMAN, S. J. (1983). Tactual roughness perception: Spatial and temporal determinants. Canadian Journal of Psychology, 37, 498-511.

Lederman, S. J., \& KLATZKY, R. L. (1987). Hand movements: A window into haptic object recognition. Cognitive Psychology, 19, 342368.

Loomis, J. M., \& Lederman, S. J. (1986). Tactual perception. In K. R. Boff, L. Kaufman, \& J. P. Thomas (Eds.), Handbook of perception and human performance: Vol. II. Cognitive processes and performance (pp. 31.1-31.41). New York: Wiley.

Miyaoka, T., Mano, T., \& OHKa, M. (1999). Mechanisms of fine surface texture discrimination in human tactile sensation. Journal of the Acoustical Society of America, 105, 2485-2492.

Morley, J. W., Goodwin, A. W., \& Darian-Smith, I. (1984). Tactile discrimination of gratings. Experimental Brain Research, 49, 291299.

Nefs, H. T., Kappers, A. M. L., \& Koenderink, J. J. (2001). Amplitude and spatial period discrimination in sinusoidal gratings by dynamic touch. Perception, 30, 1263-1274.

Rothenberg, M., Verrillo, R. T., Zahorian, S. A., Brachman, M. L., \& BolanowsKi, S. J., JR. (1977). Vibrotactile frequency for encoding a speech parameter. Journal of the Acoustical Society of America, 62, 1003-1012.

Van Boven, R. W., \& Johnson, K. O. (1994). The limit of tactile spatial resolution in humans: Grating orientation discrimination at the lip, tongue, and finger. Neurology, 44, 2361-2366.

VAN DoREN, C. L. (1989). A model of spatiotemporal tactile sensitivity linking psychophysics to tissue mechanics. Journal of the Acoustical Society of America, 85, 2065-2079.

\section{NOTES}

1. ZY-TEX paper, manufactured by Zychem Ltd. in Cheshire (U.K.). Any pattern can be printed on swell paper with an ordinary laser printer. When the printed swell paper is heated with an infrared lamp, the black areas swell; the white areas remain unchanged.

2. $\mathrm{dB}=10 *{ }^{10} \log ($ comparison/standard $)=10 *\left[{ }^{10} \log (\right.$ comparison) $-{ }^{10} \log ($ standard $\left.)\right]$.

3. Coach-Lab II was developed at the University of Amsterdam, the Netherlands. Our Coach-Lab II was slightly altered at the University of Amsterdam so that the accuracy of the ultrasonic position sensor (UPS) was higher. The maximum distance that could be measured was thereby decreased but was sufficient for our purposes.

(Manuscript received August 21, 2000; revision accepted for publication January 9, 2002.) 